\title{
DOUBLE DISTRIBUTIONS AND THE DIRICHLET PROBLEM*
}

BY

\section{O. D. KELLOGG}

In 1900 FREDHOLM published his first paper on integral equations under the title Sur une nouvelle méthode pour la résolution du problème de Divichlet. $\dagger$ He proved that if a simply connected region $R$ of the $x y$-plane is given, having a boundary curve $C$, the coördinates of whose points considered as functions of the length of arc $s$ of $C$ have continuous derivatives of third order, a potential function $u(x, y)$ of $R$ exists which has the boundary values $f(s) \ddagger$ and that it can be represented as the potential of a double distribution on $C$. Later investigations on integral equations have been concerned either with their general theory, or with their application to differential equations other than $\Delta u=0 . \S$ The object of the present paper is to establish FrEDHOLM's results with considerably lighter restrictions upon the boundary curve, and also to investigate the character of the moment of the double distribution which gives the expression for the required potential function. The results will be a broader proof of the Dirichlet principle, furnishing at the same time an expression for the required potential function and in connection with my related paper Potential Functions on the Boundary of their Region of Definition, $\|$ the establishment of simple and general conditions for the continuity at the boundary of the first derivatives of a potential function.

In the following investigation the boundary curve $C$, with coördinates $x(s)$, $y(s)$ expressed as functions of the are $s$ of $C$, will be subjected to the following condition :

(A) It shall be possible to find three positive numbers $A$, a and $\delta$

* Presented to the Society December 1, 1906. Received for publication July 18, 1907. 1900.

† Oefversigt af Kongl. Vetenskaps-Akademiens Förhandlingar, Stockholm,

† FREDHOLM makes no explicit assumptions about $f(s)$. His argument implies that $f(s)$ is in general continuous and admits an absolutely convergent integral.

\& With the exception of the work of J. PLEMEL, Über lineare Randwertaufgaben der Potentialtheorie, Monatshefte für Math. und Phys., vol. 15 (1904). He considers multiply connected regions, and reduces Fredholn's conditions upon the boundary curve to the existence of finite curvature.

$\|$ See this paper for notations and a discussion of the conditions used, page 40 of the present number of the Transactions. 
independent of $s$ and of $\Delta s$ such that $\left|x^{\prime}(s+\Delta s)-x^{\prime}(s)\right|<A|\Delta s|^{a}$, $\left|y^{\prime}(s+\Delta s)-y^{\prime}(s)\right|<A|\Delta s|^{\alpha}$, for $|\Delta s|<\delta$.

Let a potential function $u(x, y)$ be defined for the region $R$ by the values $f(s)$ which it approaches on the boundary. We seek in part $I$ to express $u(x, y)$ in the interior of $R$ as the potential of a double distribution on $C$, and proceed in part II to study the character of the moment $\phi(s)$ of this double distribution as dependent upon $f(s)$.

Part I. Expression of a potential function as the potential OF A DOUBle Distribution.

\section{§1. The functional equation.}

Let us suppose that $u(x, y)$ can be expressed by means of a double distribution,

where

$$
u(\xi, \eta)=\int_{0}^{l} \phi(t) \frac{\partial}{\partial n} \log \frac{1}{\rho} d t,
$$

$$
\rho^{2}=[\xi-x(t)]^{2}+[\eta-y(t)]^{2} .
$$

The hypothesis that î́ approaches the boundary values $\pi f(s)$-it will be convenient to introduce the factor $\pi$-leads to the functional equation for $\phi(s) *$ :

$$
f(s)=\phi(s)+\int_{0}^{l} \phi(t) \frac{1}{\pi} \frac{\partial}{\partial t}\left(\arctan \frac{y(t)-y(s)}{x(t)-x(s)}\right) d t,
$$

or, on introducing a parameter $\lambda$ and the notation

to the equation

$$
K(s, t)=\frac{1}{\pi} \frac{\partial}{\partial t}\left(\arctan \frac{y(t)-y(s)}{x(t)-x(s)}\right),
$$

$$
f(s)=\phi(s)+\lambda \int_{0}^{l} \phi(t) K(s, t) d t .
$$

This is an "integral equation of the second kind," $\dagger$ which we proceed to solve for $\phi(s)$ for the parameter value $\lambda=1$, using the methods devised by Fredholm. $\ddagger$ Before doing so however it will be necessary to consider the function $K(s, t)$ to which the name matrix of the integral equation has been given, $\S$ and to justify certain operations to be performed upon it.

* This is the usual equation of "discontinuity" for double distributions : $W_{i s}=\pi \mu_{s}+W_{\text {s. }}$ See for instance PICARD, Traité d'Analyse, vol. 2, p. 40.

†Cf. HILBERT : Grundzüge einer allgemeinen Theorie der linearen Integralgleichungen, Göttinger Nachrichten, 1904, p. 49.

I Loo. cit., and Sur une classe d'équations fonctionelles, A cta M a th e matica, vol. 27 (1903).

\$ See the abstract of Prolessor E. H. MoORE's lectures on integral equations, Yale Colloquium of the American Mathematical Society (1906). 


\section{§ 2. Operations on the matrix $K(s, t)$.}

As $K(s, t)$ has the period $l$ in both variables we may restrict ourselves to the square $0 \leqq s \leqq l, 0 \leqq t \leqq l$. If the indicated differentiation in $K(s, t)$ be carried out, this function will be found to be continuous in $s$ and $t$ except at points of the line $s=t$ and at $(0, l),(l, 0)$. Application of the law of the mean and the inequalities of condition $(A)$ show that in the neighborhood of the line $s=t$ the inequality

$$
|K(s, t)|<C_{0}|s-t|^{a-1}
$$

obtains, where $C_{0}$ and $\alpha$ are positive constants, and a similar inequality holds at the points $(0, l)$ and $(l, 0)$.

With this inequality we may prove the possibility of changing the order of certain integrations occurring in the FredHoLm method. The following equation is typical, all other interchanges being justified in the same manner:

$$
\int_{0}^{l} \int_{0}^{l} K(s, p) K(p, q) K(q, t) d p d q=\int_{0}^{l} \int_{0}^{l} K(s, p) K(p, q) K(q, t) d q d p
$$

The corners $(0, l)$ and $(l, 0)$ we shall not consider, as they present no new difficulties. Let us consider in connection with the field of integration $S$ a new field $S^{\prime}$ obtained by cutting out from $S$ strips containing the lines $p=8, p=q$, $q=t$, and bounded by the lines $p=s \pm \epsilon, p=q \pm \epsilon$, and $q=t \pm \epsilon$ respectively. The equation (3) is known to hold if the iterated integral taken in either order over the absolute value of the integrand, the field of integration being $S^{\prime}$, converges to a finite limit as $\epsilon$ approaches zero. This condition is fulfilled *; hence the equation (3) holds. Similar reasoning holds for variable limits of integration, and hence may be used to justify differentiation under an integral sign provided that the integrand, after the differentiation, satisfies inequalities of the type here used, which is always the case in what follows.

Integrals of the type (3) are of frequent occurrence in the theory of integral equations, and have received the name iterated matrices (iterierte Kerne).

* To show this, divide the square 8 into four restangles by the lines $p=(3 s+t) / 4$ and $q=(s+3 t) / 4$ and justify the equation (3) for each rectangle. Take for inetance that in which $0 \leqq p \leqq(3 s+t) / 4$, and $0 \leqq q \leqq(8+3 t) / 4$. Assume $8<t$; similar reasoning holds in the reverse case. Then $|q-t|=t-q \geqq t-(8+3 t) / 4=(t-8) / 4$, whence $|q-t|^{a-1}<|(t-8) / 1|^{a-1}$, and therefore

$\iint|K(s, p) K(p, q) K(q, t)| d q d p \leqq C_{0}^{3}|(t-8) / 4|^{a-1} \int|8-p|^{a-1} \int|p-q|^{a-1} d q d p$.

The integrals are convergent when extended ocer the strips in question and are less in value than a finite constant multiplied by $\varepsilon$ a. 
Under the hypotheses upon $K(s, t)$ they are continuous* functions of $s$ and $t$ except at points of the line $s=t$ and the points $(0, l),(l, 0)$, and this property also holds for the general iterated matrix of order $n$,

$$
K_{n}(s, t)=\int_{0}^{l} K_{n-1}(s, r) K(r, t) d r, \quad K_{0}(s, t)=K(s, t) .
$$

Moreover in the neighborhood of the line $s=t, K_{n}(s, t)$ satisfies the inequality $\dagger$

$$
\left|K_{n}(s, t)\right|<C_{n}|s-t|^{(n+1) a-1}
$$

where $C_{n}$ is a finite constant. It will be observed that as soon as we have $n>1 / \alpha-1$ the functions $K_{n}(s, t)$ are bounded.

\section{§3. The resolvent for $K(s, t)$.}

Returning now to the integral equation (1) we observe with Fredholm that there exists a function $L(s, t ; \lambda)$, called the "resolvent" for the matrix $K(s, t)$, such that the solution of $(1)$ is given by

$$
\phi(s)=f(s)-\lambda \int_{0}^{l} f(t) L(s, t ; \lambda) d t
$$

in all cases where $\lambda$ is not a zero of a certain transcendental integral function $\delta(\lambda)$. The resolvent satisfies the equations

$$
\begin{aligned}
& K(s, t)=L(s, t ; \lambda)+\lambda \int_{0}^{l} L(r, t ; \lambda) K(s, r) d r, \\
& K(s, t)=L(s, t ; \lambda)+\lambda \int_{0}^{l} L(s, r ; \lambda) K(r, t) d r .
\end{aligned}
$$

W'ere $K(s, t)$ always finite, $L(s, t ; \lambda)$ could be represented at once as the quotient of two always convergent power series in $\lambda$. In the present case, however, this is impossible and it is necessary first to find the resolvent for one of

${ }^{*}$ Given any positive number $\varepsilon$, we write $\dot{K}_{n}(s, t)=J_{1}+J_{2}$ where

and

$$
J_{1}=\int_{0}^{t-\tau}+\int_{s+\tau}^{t-\tau}+\int_{t+\tau}^{l} K_{n-1}(s, r) K(r, t) d r
$$

$$
J_{2}=\int_{s-\tau}^{s+\tau}+\int_{t-\tau}^{t+\tau} K_{n-1}(s, r) K(r, t) d r .
$$

As $s$ is not equal to $t, J_{1}$ is continuous in 8 and $t$ for fixed $\tau$, and $J_{2}$ can be made less than $\varepsilon / 4$ by restricting $\tau$ independently of $\Delta_{s}$ and $\Delta t$, provided these inorements are less than an ascertainable number. Then $\left|\Delta J_{2}\right|<\varepsilon / 2$, and because of continuity we can make $\left|\Delta J_{1}\right|<\varepsilon / 2$.

† See Fredholm, Acta Mathematica, loo. cit. The inequality holds for $n<1 / \alpha-1$. For greater values of $n$ every $K_{n}(s, t)$ is finite, as may be directly verified. If $1 / \alpha-1$ is itself an integer, we may slightly decrease $\alpha$, as this is evidently permissible. 
the finite iterated matrices $K_{n}(s, t)$, and express $L(s, t ; \lambda)$ in terms of it. To accomplish this, write

$$
\begin{gathered}
k(s, t ; \lambda)=-\left[K(s, t)+(-\lambda) K_{1}(s, t)+(-\lambda)^{2} K_{2}(s, t)\right. \\
\left.+\cdots+(-\lambda)^{n-1} K_{n-1}(s, t)\right], \\
f(s, t ; \lambda)=(-\lambda)^{n} K_{n}(s, t),
\end{gathered}
$$

where $n$ is the first odd integer greater than $1 / \alpha-1$, so that $f(s, t ; \lambda)$ is for fixed $\lambda$ a bounded function of $s$ and $t$ and is continuous except at points of the line $s=t$. For such points we may take it equal to zero inasmuch as in all applications it appears in integrals with respect to $s$ or $t$. On the other hand $k(s, t ; \lambda)$ is continuous in $s$ and $t$ except at points of the line $s=t$, in the neighborhood of which it satisfies an inequality $|k(s, t ; \lambda)|<C(\lambda)|s-t|^{a-1}$ where $C(\lambda)$ is a polynomial in $\lambda$.

The functions* $k(s, t)$ and $f(s, t)$ satisfy the equations

$$
\begin{aligned}
& f(s, t)=k(s, t)+K(s, t)+\lambda \int_{0}^{l} k(r, t) K(s, r) d r \\
& f(s, t)=k(s, t)+K(s, t)+\lambda \int_{0}^{l} k(s, r) K(r, t) d r .
\end{aligned}
$$

The resolvent for $f(s, t)$ may now be formed by the Fredholm series. Calling it $g(s, t ; \lambda)$ we have

$$
g(s, t ; \lambda)=\frac{\Gamma(s, t ; \lambda)}{\delta(\lambda)},
$$

where the transcendental integral functions $\Gamma \nmid$ and $\delta$ are given by the series

$$
\begin{aligned}
& \Gamma(s, t ; \lambda)=f(s, t ; \lambda)+\frac{\lambda}{1 !} \int_{0}^{l}\left|\begin{array}{l}
f\left(r_{1}, r_{1} ; \lambda\right), f\left(r_{1}, t ; \lambda\right) \\
f\left(s, r_{1} ; \lambda\right), f(s, t ; \lambda)
\end{array}\right| d r_{1}+\cdots
\end{aligned}
$$

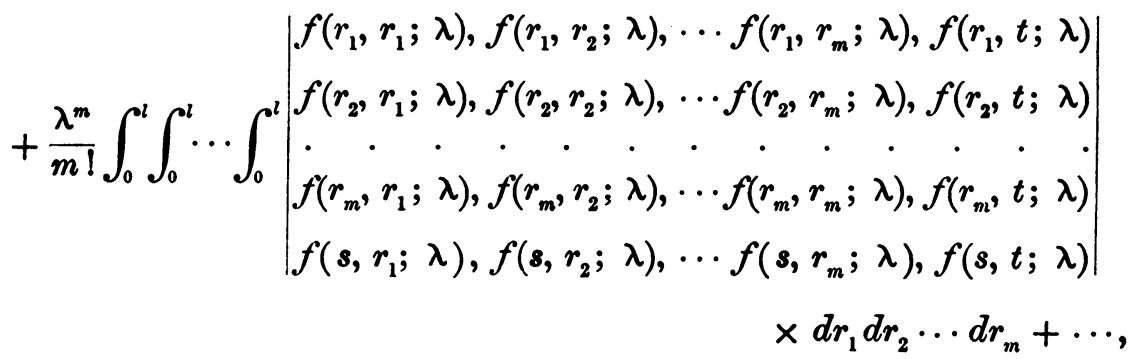

* We omit the argument $\lambda$ in the notation unless it is important to call attention to the dependence on $\lambda$.

† See the FreDHOLM convergence proof, 1. o., p. 367. 


$$
\begin{aligned}
& \delta(\lambda)=1+\frac{\lambda}{1 !} \int_{0}^{l} f\left(r_{1}, r_{1} ; \lambda\right) d r_{1}+\cdots \\
& f^{\prime}\left(r_{1}, r_{1} ; \lambda\right), f\left(r_{1}, r_{2} ; \lambda\right) \cdots f\left(r_{1}, r_{m} ; \lambda\right) \\
& +\frac{\lambda^{m}}{m !} \int_{0}^{l} \int_{0}^{l} \cdots \int_{0}^{l} f\left(r_{2}, r_{1} ; \lambda\right), f\left(r_{2}, r_{2} ; \lambda\right) \cdots f\left(r_{2}, r_{m} ; \lambda\right) d r_{1} d r_{2} \cdots d r_{m}+\cdots \\
& f\left(r_{m}, r_{1} ; \lambda\right), f\left(r_{m}, r_{2} ; \lambda\right) \cdots f\left(r_{m}, r_{m} ; \lambda\right)
\end{aligned}
$$

The quotient of (10) by (11) is also given by the series*

$$
g(s, t ; \lambda)=f(s, t ; \lambda)-\lambda f_{1}(s, t ; \lambda)+\lambda^{2} f_{2}(s, t ; \lambda) \cdots+(-\lambda)^{m} f_{m}(s, t ; \lambda)+\cdots
$$
where

$$
f_{m}(s, t ; \lambda)=\int_{0}^{l} f_{m-1}(s, r ; \lambda) f(r, t ; \lambda) d r, \quad\left(f_{0}=f\right) .
$$

Remembering the definition of $f(s, t ; \lambda)$ in equation (8) we see that (10) and $(11) *$ are uniformly convergent for any fixed $\lambda$, and that (12) is uniformly convergent for any $\lambda$ less in absolute value than $1 / \sqrt[n+1]{K l}$ where $K$ is the maximum of $\left|K_{n}(s, t)\right|$. It follows that these three series are continuous functions of $s$ and $t$, except at points of the line $s=t$, and that they are bounded. The function $g(s, t)$ satisfies the equations

$$
\begin{aligned}
& f(s, t)=g(s, t)+\lambda \int_{0}^{l} g(r, t) f(s, r) d r, \\
& f(s, t)=g(s, t)+\lambda \int_{0}^{t} g(s, r) f(r, t) d r .
\end{aligned}
$$

It is now only necessary to eliminate $f(s, t)$ between (13) and (9). Suppose the value of $f(s, t)$ be substituted from $\left(9_{1}\right)$ into $\left(13_{1}\right)$, denoting by the subscript the first equation in each set. We obtain, if we properly change the notation for the variables of integration,

$$
\begin{aligned}
K(s, t) & =-k(s, t)+g(s, t)+\lambda \int_{0}^{l} g(q, t) k(s, q) d q \\
& +\lambda \int_{0}^{l}\left\{-k(r, t)+g(r, t)+\lambda \int_{0}^{l} g(q, t) k(r, q) d q\right\} K(s, r) d r .
\end{aligned}
$$

Accordingly if we write

$$
L(s, t ; \lambda)=-k(s, t ; \lambda)+g(s, t ; \lambda)+\lambda \int_{0}^{l} g(q, t ; \lambda) k(s, q ; \lambda) d q,
$$

we are led to equations $(6), \dagger$ which show the $L(s, t ; \lambda)$ thus obtained to be a resolvent for $K(s, t)$.

* See my note in Göttinger Nachrichten for 1902, p. 165.

† The second equation $\left(6_{2}\right)$ is similarly derived from $\left(9_{2}\right)$ and $\left(13_{2}\right)$. It is to be noted, how ever, that if we are to obtain the same $L(s, t ; \lambda)$ in the second equation, we must have

$$
\int_{0}^{t} g(s, q) k(q, t) d q=\int_{0}^{t} g(q, t) k(s, q) d q .
$$


§4. Solution of the functional equation.

Using the resolvent in the form

$$
\begin{aligned}
L(s, t ; \lambda) & =-k(s, t ; \lambda)+-\frac{\Gamma(s, t ; \lambda)+\lambda \int_{0}^{t} \Gamma(r, t ; \lambda) k(s, r ; \lambda) d r}{\delta(\lambda)} \\
& =-k(s, t ; \lambda)+\frac{\Gamma(s, t ; \lambda)+\lambda \int_{0}^{t} \Gamma(s, r ; \lambda) k(r, t ; \lambda) d r}{\delta(\lambda)}
\end{aligned}
$$

we attack the problem of solving the equation (1).

Let us denote by $m$ the order of $\lambda=1$ as a zero of $\delta(\lambda)$; in particular, $m$ may be zero. Let $m^{\prime}$ be the index of the highest power of $\lambda-1$ that divides

$$
\Gamma(s, t ; \lambda)+\lambda \int_{0}^{l} \Gamma(s, r ; \lambda) k(r, t ; \lambda) d r,
$$

which we denote by $N(s, t ; \lambda)$, so that

$$
\left.\frac{N(s, t ; \lambda)}{(\lambda-1)^{m^{\prime}}}\right|_{\lambda=1}
$$

is neither infinite nor identically zero. We assume now that $m \leqq m^{\prime}$ so that $\lambda=1$ is not a pole of $L(s, t ; \lambda)$, and hence $L(s, t ; 1)$ is defined ; it will be a continuous function of $s$ and $t$ except at points of the line $s=t$, where it satisfies an inequality of the same form as that to which $k(s, t ; 1)$ is subjected. We may now solve the equation (1) if we place upon $f(s)$ the restriction that it shall be integrable and shall admit the changes of order of integration occurring in the following.* Putting $\lambda=1$ in equation (1), multiplying by $L(q, s ; 1) d s$ and integrating; then applying $\left(6_{2}\right)$ for $\lambda=1$, we have

$$
\begin{aligned}
\int_{0}^{l} f(s) L(q, s ; 1) d s=\int_{0}^{l} \phi(s) L(q, s ; 1) d s \\
+\int_{0}^{l} \int_{0}^{l} \phi(t) K(s, t) L(q, s ; 1) d t d s=\int^{l} \phi(t) K(q, t) d t,
\end{aligned}
$$

and this, by (1), is $f(q)-\phi(q)$, so that,

$$
\phi(s)=f(s)-\int_{0}^{l} f(t) L(s, t ; 1) d t .
$$

This equation is easily verified by nsing the series (12) and remembering that the order of integrations involved may be changed.

* Such will be the case if $f(s)$ is continuous except at isolated points $s_{i}$ in the neighborhood of which $|f(s)|<B\left|s-s_{i}\right| \beta-1$, where $B$ and $\beta$ are positive constants. 
If therefore (1) has a solution admitting the changes of order of integration involved, it must be given by (5); and conversely, the value of $\phi(8)$ in (5) satisfies (1), as may be verified by direct substitution and use of equation $\left(6_{1}\right)$. Thus, on the assumption that $\lambda=1$ is not a pole of $L(s, t ; \lambda)$, we have obtained the solution of (1) and shown that the potential function $u(x, y)$ is expressible as the potential of a double distribution on $C$. Moreover the solution $\phi(s)$ is unique among all functions admitting the integrations and changes of order. For the difference of two solutions, $\phi_{1}(s)-\phi_{2}(s)=\psi(s)$, would satisfy the equation

$$
0=\psi(s)+\int_{0}^{l} \psi(t) K(s, t) d t,
$$

and the process which derived (5) from (1), if applied to this equation, leads to the conclusion $\psi(s)=0$. It now only remains to justify the assumption made, and show that $m \leqq m^{\prime}$.

Supposing the contrary to be the case, that is, $m^{\prime}<m$, the function

$$
\Phi(s, t)=\left.\frac{N(s, t ; \lambda)}{\delta(\lambda)}(\lambda-1)^{m-m^{\prime}}\right|_{\lambda=1}
$$

is not identically zero; it is bounded, and also continuous except possibly at points of the line $s=t$. Multiplying $\left(6_{1}\right)$ by $(\lambda-1)^{m-m^{\prime}}$ and putting $\lambda=1$ we have

$$
0=\Phi(s, t)+\int_{0}^{l} \Phi(r, t) \bar{X}(s, r) d r .
$$

Integrating this with respect to $t$ from 0 to $t$, and denoting

by $\Psi(s, t)$ we have

$$
\int_{0}^{t} \Phi(s, t) d t
$$

$$
0=\Psi(s, t)+\int_{0}^{t} \Psi(r, t) K(s, r) d r .
$$

But this admits the interpretation: $\Psi(s, t)$ is for any fixed $t$ the moment of a double distribution whose potential $W$ vanishes within $R$. Its normal derivatives therefore also vanish, but in case the moment of a double distribution satisfies condition $(B)^{*}$, its normal derivatives join on continuously to those of the potential $W^{\prime}$ of the same double distribution in the exterior region $R^{\prime}$. Hence on applying Green's formula, $W^{\prime}$ is constant; but as it vanishes at $\infty$, $W^{\prime}=0$. Finally, as the moment of the double distribution multiplied by $2 \pi$ is the difference of the boundary values of $W$ and $W^{\prime}$, we have $\Psi(s, t)=0$,

* This condition is stated in the next paragraph. That it is satisfied by $\Psi(s, t)$ will be shown later. For the theorem stated concerning the normal derivatives of a double distribution see my paper Potential functions, previously cited, p. 44. 
whence $\Phi(s, t)=0$. This proves the falsity of the assumption $m^{\prime}<m$. We may therefore state the following theorem :

Given a function $f(s)$ of the arc of $C$, continuous except at isolated points $s_{i}$ in the neighborhood of which $|f(s)|<B\left|s-s_{i}\right|^{\beta-1}$, where $B$ and $\beta$ are positive constants; there exists a potential function $u(x, y)$ of $R$ which approaches $f(s)$ at all points where $f(s)$ is continuous, and $u(x, y)$ may be represented in the interior of $R$ as the potential of a double distribution on $C$; * moreover the double distribution is uniquely determined by the demand that its moment satisfy the same continuity requirements as $f(s)$.

\section{Part II. The character of the moment $\phi(8)$.}

In my paper cited above it was shown that a potential function $u(x, y)$ of $R$ which coincides within $R$ with the potential of the double distribution on $C$,

$$
W(\xi, \eta)=\int_{0}^{l} \phi(t) \frac{\partial}{\partial n} \log \frac{1}{\rho} d t,
$$

and which is defined on the boundary by its limiting values, has continuous first derivatives in the closed region $R$ provided the boundary $C$ satisfies conditions $(A)$, and $\phi(s)$ satisfies the following condition:

(B) The function $\phi^{\prime}(\mathrm{s})$ is continuous, and the integral

$$
\int_{0}^{\tau}\left|\frac{\phi^{\prime}(s+t)-\phi^{\prime}(s-t)}{t}\right| d t
$$

approaches 0 uniformly with respect to s as $\tau$ approaches 0 .

It was further stated, the proof being deferred to the present paper, that if $u(x, y)$ is defined by boundary values $f(s)$ satisfying condition $(B)$, it can be represented as the potential of a double distribution on $C$; and moreover that the moment $\phi(s)$ of the double distribution satisfies the same condition $(B)$. We have now justified the first part of this statement, and it remains to prove the part concerning the moment. At the same time we shall see that the function $\Psi(s, t)$ for fixed $t$ has the same character as $\phi(s)$, a fact essential to the reasoning in the preceding page.

A study of equation (5) and of the function $L(s, t ; \lambda)$ reveals the continuity of $\phi^{\prime}(s)$ and $\Psi_{,}^{\prime}(s, t)$ (with possible exception of isolated points, near which the functions remain finite); by means of this fact and equations (1) and $(6)$, we shall show that $\phi(s)$ and $\Psi(s, t)$ satisfy $(B)$.

\footnotetext{
* The generality of this as an existence proof depends upon the lightness of condition ( 4 ).
} 


\section{§1. Continuity of the functions involved.}

It is not evident that the equation (5) can be differentiated with respect to 8, a process necessary to the study of $\phi^{\prime}(s)$. Its form must first. be changed by integration by parts. In doing this we meet with the function

$$
\frac{\partial}{\partial s} \int_{0}^{t} L(s, t ; \lambda) d t,
$$

which must be investigated first.

Let us suppose that we are measuring $s$ from a point where the tangent to $C$ is parallel with the $x$-axis. Then the function

$$
\Theta(s, t)=\frac{1}{\pi} \arctan \frac{y(s)-y(t)}{x(s)-x(t)}
$$

may be made one-valued in the region $0 \leqq s<l, 0 \leqq t<l$ by the demand that it pass continuously over into 0 as $s$ and $t$ approach $00^{*}$ We have then the following relations : $\dagger$

$$
\begin{gathered}
\Theta(s, t)=\Theta(t, s), \quad \Theta(s, l)-\Theta(s, 0)=\Theta(l, t)-\Theta(0, t)=1 \\
\frac{\partial}{\partial t} \Theta(s, t)=K(s, t), \quad \frac{\partial}{\partial s} \Theta(s, t)=K(t, s) \\
\int_{0}^{t} K(s, t) d t=\Theta(s, t)-\Theta(s, 0), \quad \int_{0}^{l} K(s, t)=1 \\
\frac{\partial}{\partial s} \int_{0}^{t} K(s, t) d t=K(t, s)-K(0, s) .
\end{gathered}
$$

We seek an equation similar to the last for

$$
\frac{\partial}{\partial 8} \int_{0}^{t} L(s, t ; \lambda) d t .
$$

Defining the continuous $\ddagger$ function

we find that

$$
\Theta_{1}(s, t)=\int_{0}^{t} K(s ; r) \Theta(r, t) d t
$$

and

$$
\Theta_{1}(s, l)-\Theta_{1}(s, 0)=1, \quad \Theta_{1}(l, t)-\Theta_{1}(0, t)=0,
$$

$$
\frac{\partial}{\partial t} \Theta_{1}(s, t)=K_{1}(s, t) \text {. }
$$

* AMms, Note on the orientation of a secant, Bulletin of the Amerioan Mathematioal Sooiety, vol. 13 (1907), p. 240.

$\dagger$ It is to be nnderstood that $\theta(s, l)=\lim _{t=l} \theta(s, t), \quad \theta(l, t)=\lim _{s=l} \theta(s, t)$.

¥ That $\theta_{1}(8, t)$ is continuous may be proven as in the footnote, p. 54 . 
To obtain $\partial \Theta_{1}(s, t) / \partial s$ integrate first by parts in the equation defining $\Theta_{1}(s, t)$. The result is

whence

$$
\Theta_{1}(s, t)=1+\Theta(s, 0)+\Theta(0, t)-\int_{0}^{t} \Theta(s, r) K(t, r) d r
$$

and hence

$$
\begin{gathered}
\frac{\partial}{\partial s} \Theta_{1}(s, t)=K(0, s)-K_{1}(t, s) \\
\int_{0}^{t} K_{1}(s, t) d t=\Theta_{1}(s, t)-\Theta_{1}(s, 0),
\end{gathered}
$$

$$
\frac{\partial}{\partial s} \int_{0}^{t} K_{1}(s, t) d t=-\left[K_{1}(t, 8)-K_{1}(0,8)\right] .
$$

Proceeding in a similar manner we obtain by mathematical induction the formula for the general iterated matrix :

$$
\frac{\partial}{\partial s} \int_{0}^{t} K_{i}(s, t) d t=(-1)^{i}\left[K_{i}(t, s)-K_{i}(0, s)\right] \text {. }
$$

With these results equations (7), (8) and (12) give

$$
\begin{aligned}
& \frac{\partial}{\partial s} \int_{0}^{t} k(s, t ; \lambda) d t=k(t, s ;-\lambda)-k(0, s ;-\lambda), \\
& \frac{\partial}{\partial s} \int_{0}^{t} f(s, t ; \lambda) d t=f(t, s ;-\lambda)-f(0, s ;-\lambda), \\
& \frac{\partial}{\partial s} \int_{0}^{t} g(s, t ; \lambda) d t=g(t, s ;-\lambda)-g(0, s ;-\lambda),
\end{aligned}
$$

and as $\delta(\lambda)=\delta(-\lambda)$,

$$
\frac{\partial}{\partial s} \int_{0}^{t} \Gamma(s, t ; \lambda) d t=\Gamma(t, s ;-\lambda)-\Gamma(0, s ;-\lambda) .
$$

The analogous equation holds for the function

$$
\lambda \int_{0}^{l} \Gamma(r, t ; \lambda) k(s, r ; \lambda) d r \equiv \lambda \delta(\lambda) \int_{0}^{l} g(r, t ; \lambda) k(s, r ; \lambda) d r,
$$

whence by (15) the desired result,

$$
\frac{\partial}{\partial s} \int_{0}^{t} L(s, t ; \lambda) d t=L(t, s ;-\lambda)-L(0, s ;-\lambda)
$$

is obtained.

* Equations (8) and (11) show $\delta(\lambda)$ to be a power series in $\lambda^{n+1}$; the grounds for taking $n$ odd (p. 55) are thus apparent. 
Finally we must consider for $m^{\prime}<m$ the expression

$$
\left[L(s, t, \lambda)(\lambda-1)^{m-m^{\prime}}\right]_{\lambda=1}=\Phi(s, t) .
$$

The development of the integral transcendental functions involved about the point $\lambda=1$ leads to the result

$$
\begin{aligned}
\frac{\partial}{\partial s} \int_{0}^{t}\left[L(s, t ; \lambda)(\lambda-1)^{m-m^{\prime}}\right]_{\lambda=1} d t \\
\quad=\left[\{L(t, s ;-\lambda)-L(0, s ;-\lambda)\}(\lambda-1)^{m-m^{\prime}}\right]_{\lambda=1} .
\end{aligned}
$$

This function, which is none other than $\Psi_{s}^{\prime}(s, t)$, appears as a uniformly convergent series of iterated matrices, all of order $n$ or higher. It is therefore continuous except possibly at points of the lines $s=t$ and $s=0$, in the neighborhood of which it is finite, so that we may state the result:

$\Psi_{s}^{\prime}(s, t)$ is bounded, and for fixed $t$ it is a continuous function of $s$ except possibly at the points $s=t$ and $s=0$.

In the case $m \leqq m^{\prime}$, for which $\lambda=1$ is not a pole of $L(s, t ; \lambda)$, we may use equation (17) for $\lambda=1$. Then integrating by parts in equation (5) we have

$$
\begin{aligned}
\phi(s) & =f(s)-\left[f(t) \int_{0}^{t} L(s, t ; 1) d t\right]_{t=0}^{t=l}+\int_{0}^{l} f^{\prime \prime}(t) \int_{0}^{t} L(s, t ; 1) d t \\
& =f(s)-f(l) \int_{0}^{l} L(s, t ; 1) d t+\int_{0}^{l} f^{\prime \prime}(t) \int_{0}^{t} L(s, t ; 1) d t .
\end{aligned}
$$

If now the equation $\left(6_{2}\right)$ for $\lambda=1$ be integrated with respect to $t$ from 0 to $l$, we have by (17),

$$
\int_{0}^{l} L(s, t ; 1) d t=\frac{1}{2} \cdot *
$$

Hence differentiating the above equation for $\phi(s)$, we obtain

$$
\begin{aligned}
\phi^{\prime}(s) & =f^{\prime}(s)+\int_{0}^{l}\left[f^{\prime}(t) \frac{\partial}{\partial s} \int_{0}^{t} L(s, t ; 1) d t\right] d t \\
& =f^{\prime \prime}(s)+\int_{0}^{l} f^{\prime}(t) L(t, s ;-1) d t-L(0, s ;-1) \int_{0}^{l} f^{\prime}(t) d t \\
& =f^{\prime}(s)+\int_{0}^{l} f^{\prime}(t) L(t, s ;-1) d t,
\end{aligned}
$$

which proves that $\phi^{\prime}(s)$ is continuous. $\dagger$

* It is interesting to note that this equation precludes the possibility $m<m^{\prime}$. For then $L(s, t ; 1)=-k(s, t ; 1)$, and from the definition of $k(s, t ; \lambda)$, we see that

is an integer.

$$
\int_{0}^{l} k(s, t ; 1) d t
$$

The development of the integral transcendental functions involved about $\lambda=1$, shows that $L(t, 8 ;-1)$ is equal to $-k(t, 8 ;-1)$ plus a uniformly convergent series of iterated matrices 
§2. The functions $\phi(s)$ and $\Psi(s, t)$ satisfy conditions $(B)$.

Integrating by parts in equations (1) and (16) and then differentiating with respect to 8 , we have

$$
\begin{aligned}
f^{\prime}(s) & =\phi^{\prime}(s)-\int_{0}^{l} \phi^{\prime}(t) K(t, s) d t, \\
0 & =\psi_{0}^{\prime}(s, t)-\int_{0}^{l} \psi_{r}^{\prime}(r, t) K(r, s) d r .
\end{aligned}
$$

If now two functions satisfy condition $(B)$, their difference does. Hence as $f(s)$ does, it is only necessary to show that the two integrals of the last equations do. This is included in the following theorem which we proceed to prove.

If $g(s)$ is bounded, and is continuous except at isolated points, the function

$$
I(s)=\int_{0}^{l} g(t) K(t, s) d t
$$

where $K(t, s)$ is the function defined above, is uniformly algebraically continuous, that is, satisfies the hypothesis upon $x^{\prime}(s)$ and $y^{\prime}(s)$ in condition $(A)$.

For convenience let us take as origin for the variable $s$ the point to be considered. We are to show that we can determine three positive constants, $C^{\prime}, \alpha^{\prime}$, and $\delta^{\prime}$, independent of $s$ and $\Delta s$, such that

$$
|I(s+\Delta s)-I(s)|<C^{\prime}|\Delta s|^{a^{\prime}}
$$

as soon as $|\Delta s|<\delta^{\prime}$; or in particular, for the point in question,

$$
|I(s)-I(0)|<C^{\prime}|s|^{a^{\prime}}
$$

as soon as $|s|<\delta^{\prime}$. To do this, write $I=I_{1}+I_{2}+I_{3}$, where

and

$$
I_{1}=\int_{-\sqrt{s}}^{\sqrt{s}} g(t) K(t, s) d t, \quad I_{2}=\int_{-\eta}^{-\sqrt{s}}+\int_{\sqrt{s}}^{\eta} g(t) K(t, s) d t,
$$

$$
I_{3}=\int_{-\frac{l}{2}}^{\cdot-\eta}+\int_{\eta}^{\frac{l}{2}} g(t) K(t, s) d t,
$$

leaving the number $\eta$ to be specified presently. We have supposed $s \geqq 0$; the reasoning for $s \leqq 0$ is exactly similar.

Considering $I_{1}(s)$ with the inequality (2) for $K(t, s)$, we have

of order $n$ or higher; that is, plus a function of 8 and $t$ which is continuous except possibly at points of the line $s=t$, in the neighborhood of which it is bounded. Reasoning like that of the footnote, p. 54, then demonstrates the asserted continuity of $\phi^{\prime}(8)$. 


$$
\left|I_{1}(s)\right|<\max |g(t)| C_{0} \int_{-\sqrt{t}}^{\sqrt{t}}|s-t|^{a-1} d t<C_{1}^{\prime} s^{\frac{\alpha}{2}}
$$

where $C_{1}^{\prime}$ is a finite constant. Then as $I_{1}(0)=0$, we have

$$
\left|I_{1}(8)-I_{1}(0)\right|<C_{1}^{\prime} s^{\frac{a}{2}}
$$

For $I_{2}(s)$ we have

$$
\begin{aligned}
I_{2}(s)-I_{2}(0) & =\int_{-\eta}^{-\sqrt{t}}+\int_{\sqrt{s}}^{\eta} g(t) K(t, s) d t-\int_{-\eta}^{\eta} g(t) K(t, 0) d t \\
& =\int_{-\eta}^{-\sqrt{t}}+\int_{\sqrt{t}}^{\eta} g(t)[K(t, s)-K(t, 0)] d t-\int_{-\sqrt{8}}^{\sqrt{s}} g(t) K(t, 0) d t,
\end{aligned}
$$

where the last integral satisfies the above inequality for $\left|I_{1}(s)\right|$. Hence

$$
\left|I_{2}(s)-I_{2}(0)\right|<\max |g(t)|\left\{\int_{-\eta}^{-\sqrt{t}}+\int_{\sqrt{s}}^{\eta}|K(t, s)-K(t, 0)| d t\right\}+C_{1}^{\prime} s^{\frac{a}{2}}
$$

The sum of the two integrals, in which $\sqrt{s} \leqq|t| \leqq \eta$, will be found less than a finite constant times $8^{a / 2}$. To show this we study the common integrand, writing

$$
\begin{gathered}
K(t, s)-K(t, 0)=N(t, s) / \pi \rho^{2} \rho_{0}^{2}, \\
\rho^{2}=[x(t)-x(s)]^{2}+[y(t)-y(s)]^{2}, \quad \rho_{0}^{2}=[x(t)-x(0)]^{2}+[y(t)-y(0)]^{2}, \\
\begin{array}{r}
N(t, s)=\rho_{0}^{2}\left\{[x(s)-x(t)] y^{\prime}(s)-[y(s)-y(t)] x^{\prime}(s)\right\} \\
-\rho^{2}\left\{[x(0)-x(t)] y^{\prime}(0)-[y(0)-y(t)] x^{\prime}(0)\right\} .
\end{array}
\end{gathered}
$$

The law of the mean applied to the differences involved gives $\rho^{2}=(s-t)^{2} F(s, t)$, $\rho_{0}^{2}=t^{2} F(0, t)$, where $F(s, t)$ has a positive lower limit $\underline{F}$, so that $\rho^{2} \geqq(s-t)^{2} \underline{F}$ and $\rho_{0}^{2} \geqq t^{2} \underline{F}$. To obtain therefore an effective upper limit for the integral of (22) we must arrange $N(t, 8)$ so as to expose the factors $(s-t), s, t$ or fractional powers of them. We may do this by writing $N=N_{1}+N_{2}+N_{3}$, where

$$
\begin{aligned}
& N_{1}=\rho_{0}^{2}\left\{\left[y^{\prime}(s)-y^{\prime}(0)\right][x(s)-x(t)]-\left[x^{\prime}(s)-x^{\prime}(0)\right][y(s)-y(t)]\right\}, \\
& N_{2}=\rho_{0}^{2}\left\{[x(s)-x(0)] y^{\prime}(0)-[y(s)-y(0)] x^{\prime}(0)\right\}, \\
& N_{3}=\left(\rho_{0}^{2}-\rho^{2}\right)\left\{[x(0)-x(t)] y^{\prime}(0)-\left[y^{2}(0)-y(t)\right] x^{\prime}(0)\right\} .
\end{aligned}
$$

The law of the mean together with condition $(A)$ leads, provided $2 \eta$ is less than $\delta$ of that condition, to the equations 


$$
\begin{array}{ll}
x^{\prime}(s)-x^{\prime}(0)=F \cdot s^{a}, & y^{\prime}(s)-y^{\prime}(0)=F \cdot s^{a}, \\
x(s)-x(t)=F \cdot(s-t), & y(s)-y(t)=F \cdot(s-t), \\
x(s)-x(0)=s\left[x^{\prime}(0)+F \cdot s^{a}\right], & y(s)-y(0)=s\left[y^{\prime}(0)+F^{\prime} \cdot s^{a}\right],
\end{array}
$$

where $F$ denotes various bounded functions. These give

$$
\begin{gathered}
\qquad N_{1}=\rho_{0}^{2} F \cdot s^{a}|s-t|, \quad N_{2}=\rho_{0}^{2} F \cdot s^{a+1}, \\
\rho_{0}^{2}-\rho^{2}=-[x(s)-x(0)][x(s)+x(0)-2 x(t)] \\
\text { whence } \quad-[y(s-y(0)][y(s)+y(0)-2 y(t)]=F \cdot s\{|s-t|+|t|\}, \\
N_{3}=F \cdot s|t|^{a+1}\{|s-t|+|t|\} .
\end{gathered}
$$

Using these values and denoting by $\bar{F}$ a constant greater than any $F / \underline{F}$, we have

$$
|K(t, s)-K(t, 0)|<\overline{F^{2}} s^{\frac{a}{2}}\left\{s^{\frac{a}{2}}\left(\frac{1}{|s-t|}+\frac{s}{(s-t)^{2}}\right)+s^{1-\frac{a}{2}}|t|^{a-1}\left(\frac{1}{|s-t|}+\frac{|t|}{(s-t)^{2}}\right)\right\} \text {. }
$$

The quantity in braces will be found less than a lnear function of $|t|^{a-1}$. For $|t| \geqq \sqrt{s}$, whence $|t| \geqq t^{2} \geqq s$ if $|t|<1$, which we can effect by choosing $\eta \leqq 1$; hence $|s-t| \geqq|t|-s \geqq|t|-t^{2}=|t|(1-|t|)$. Further, on restricting $\eta$ to be less than $\frac{1}{2}$, this gives $|s-t|>|t| / 2$. Whence

Similarly

$$
\frac{8^{\frac{a}{2}}}{|s-t|}<\frac{28^{\frac{a}{2}}}{|t|} \leqq \frac{2|t|^{a}}{|t|}=2|t|^{a-1} .
$$

Hence,

$$
\frac{s^{1+\frac{a}{2}}}{|s-t|^{2}}<4 t^{\alpha}, \quad \frac{s^{1-\frac{a}{2}}|t|^{a-1}}{|s-t|}<2, \quad \frac{s^{1-\frac{a}{2}}|t|^{a}}{|s-t|^{2}}<4 .
$$

$$
|K(t, s)-K(t, 0)|<\overline{F^{3}} s^{\frac{a}{2}}\left\{10+2|t|^{a-1}\right\} .
$$

With this the inequality (22) becomes

$$
\left|I_{2}(s)-I_{2}(0)\right|<C_{2}^{\prime} s^{\frac{a}{2}} \text {. }
$$

Finally, consider $I_{3}(s)$. We restrict $\delta$ to be less than $\eta / 2$ and hence it follows that $|s-t|>\eta / 2,|t|$ being greater than $\eta$, so that the rational function $K(t, s)$ of uniformly algebraically continuous functions of $s$ has a denominator with positive lower limit, and is hence itself uniformly algebraically continuous with the same index $\alpha$. Consequently for suitably chosen $C_{3}^{\prime}$,

$$
\left|I_{3}(s)-I_{3}(0)\right|<C_{3}^{\prime} s^{a} \leqq C_{3}^{\prime s^{\frac{a}{2}}}
$$


From the inequalities (21), (23) and (24) there results the inequality

$$
|I(s)-I(0)|<C^{\prime} s^{a^{\prime}},
$$

in which $C^{\prime}=C_{1}^{\prime}+C_{2}^{\prime}+C_{3}^{\prime}$ and $\alpha^{\prime}=\alpha / 2$, where $C^{\prime}$ as well as $\alpha^{\prime}$ and $\delta^{\prime}$ are independent of the particular point $s$ investigated. Accordingly we may write the inequality

$$
|I(s+\Delta s)-I(s)|<C^{\prime}|\Delta s|^{a^{\prime}}
$$

as obtaining for every $s$, as soon as $|\Delta s|<\delta^{\prime}$, and the above stated theorem concerning $I(s)$ is established.

Thus $\Psi(s, t)$ for fixed $t$ satisfies condition $(B)$, and the assumption of page 58 is justified, and consequently the case for which $\lambda=1$ is a pole of $L(s, t ; \lambda)$ is impossible. For $\phi(s)$ we may state the theorem :

If the boundary values $f(s)$ of a potential function $u(x, y)$ satisfy condition $(B)$, so also does the moment $\phi(8)$ of the double distribution on $C$ whose potential coincides with $u(x, y)$ within $R$.

Columbia, Missouri,

July, $190 \%$. 